\title{
Comment le marketing politique et publicitaire construit la mythologie de la diversité
}

\section{Marie-Cécile Naves}

\section{(2) OpenEdition \\ 1 Journals}

Édition électronique

URL : http://journals.openedition.org/mots/20634

DOI : $10.4000 /$ mots. 20634

ISSN : 1960-6001

Éditeur

ENS Éditions

\section{Édition imprimée}

Date de publication : 1 mai 2012

Pagination : 95-102

ISBN : 978-2-84788-356-5

ISSN : 0243-6450

\section{Référence électronique}

Marie-Cécile Naves, «Comment le marketing politique et publicitaire construit la mythologie de la diversité ", Mots. Les langages du politique [En ligne], 98 | 2012, mis en ligne le 01 mai 2014, consulté le 19 avril 2019. URL : http://journals.openedition.org/mots/20634 ; DOI : 10.4000/mots.20634

\section{(c) ENS Éditions}




\section{Comment le marketing politique et publicitaire construit la mythologie de la diversité}

Valoriser ou, plus rarement, critiquer la « diversité » constitue aujourd'hui deux biais majeurs du marketing politique comme publicitaire occidental. S'inscrivant dans la lutte contre les discriminations (Fassin et Fassin, 2006) ou jouant, au contraire, par le rejet du métissage et le repli sur la «société fermée » (Perrineau, 2001), sur les peurs que ce mouvement suscite, la communication s'est emparée de cette importante évolution. La diversité est devenue un instrument du libéralisme économique et politique. Elle fait alors figure de storytelling, autrement dit de récit collectif, porteur d'une idéologie qui « constitue une réponse à la crise du sens [...] et un outil de propagande» (Salmon, 2008, p. 13). Cet « art de raconter des histoires», à partir d'une évolution sociale réelle mais mythifiée et mystifiée du vivre-ensemble, est mis au service de finalités commerciales ou politiques, et constitue en cela une forme d'aliénation, la diversité étant présentée comme étant, en soi et $a$ priori, source de richesse ou a contrario d'appauvrissement de la société française.

Il s'agit tout d'abord de déconstruire la notion de diversité pour en comprendre les origines et les conditions d'émergence dans l'espace politique, économique et médiatique français. Ensuite, nous nous efforcerons, grâce aux outils de l'analyse de discours, d'étudier comment et en quoi, en tant que fantasme collectif, elle est devenue un ressort du marketing par une construction de discours qui se font l'écho des structures sociales d'une époque (Foucault, 1969). Les évolutions du vocabulaire peuvent en effet être tout autant un révélateur qu'un vecteur du changement social, du fait de leur appropriation par les acteurs politiques et économiques.

Nous verrons, à travers quelques exemples emblématiques de discours sur la diversité, que les registres rhétoriques du plaisir, de la morale, de la performance, de l'harmonie ou au contraire du danger et de la violence sont tour à 
tour mobilisés et que les stratégies narratives sont politiques dans la publicité, et publicitaires dans le politique.

\section{Contexte d’apparition de la notion de diversité}

L'analyse des discours est inséparable de leur contexte d'énonciation, c'està-dire des faits et des représentations collectives propres à ce dernier. Ainsi, la «diversité », expression polysémique apparue en France à la charnière des années 1990 et 2000, synthétise, sans doute de manière euphémisée, l'idée de pluralisme des cultures, des origines "ethniques», voire "raciales», des religions, des genres et des orientations sexuelles. L'effritement des grandes idéologies et les débats occasionnés par le souci d'une meilleure représentativité publique et d'un meilleur accès aux ressources des femmes et des minorités sont les principales raisons de cette évolution. Sur les plans juridique et administratif, certaines instances internationales ont pour leur part donné à la notion de diversité culturelle une connotation positive, porteuse de paix et de tolérance. Ainsi l'Unesco validait-elle, en 2001, une «Déclaration universelle sur la diversité culturelle», érigeant les différences de traditions et de contenus culturels en «héritage commun » et participant du « respect de la dignité humaine ${ }^{1}$. De son côté, l'Union européenne a fait de la promotion de la diversité un outil de lutte contre le racisme et le sexisme, mais aussi un facteur de rapprochement des peuples et de renforcement de l'identité de l'Europe.

En France, jusqu'aux années 1990, le modèle universaliste et laïc n'avait que peu été remis en cause : le prisme des inégalités de classe et le cadre républicain de neutralité vis-à-vis de la religion, de l'origine ou du genre demeuraient dominants. La prise en compte de la diversité était même essentiellement vue comme une menace pour l'unité de la nation. Comme l'a expliqué Éric Fassin (2002), les débats sur le Pacte civil de solidarité et sur la parité ont, dans une France «réputée antiminoritaire» et brandissant «l'épouvantail du politiquement correct américain », introduit une rupture et largement contribué à la mutation de certains cadres d'analyse de la société. En effet, l’interrogation conjointe sur la représentation politique et sur la discrimination ne s'est pas limitée aux seules questions de genre et d'orientation sexuelle, mais «s'est répercutée dans le domaine de l'ethnicité », que ce soit par le biais légal (loi du 16 novembre 2001, notamment) ou la mise en place d'organismes publics, à l'instar de la Haute autorité de lutte contre les discriminations et pour l'égalité (Halde), créée en 2004. Le combat contre les discriminations s'est voulu plus efficace et a complexifié la problématique des inégalités sociales. Conjointe-

1. L'importance accordée, depuis les années 1970, à l’impératif de «dialogue des cultures » dans la francophonie a inspiré cette Déclaration de l’Unesco. 
ment, le vote de lois mémorielles, entre 1990 et 2005, marque la reconnaissance symbolique (et pénale), par la République, d'un pluralisme de cultures et de religions dotées d'histoires spécifiques. Du côté de la société civile, des associations communautaires, comme par exemple le CRAN (Conseil représentatif des associations noires) en 2005, ont vu le jour pour à la fois dénoncer le racisme anti-Noirs et donner plus de visibilité à la «culture noire».

C'est dans ce contexte, qui mêle enjeux de redistribution et de reconnaissance ${ }^{2}$, que vont se construire diverses mythologies de la diversité.

\section{Tous différents mais tous égaux, voilà notre richesse, selon la publicité}

Le monde économique s'est inscrit dans ce mouvement de fond par l'élaboration de diverses stratégies de communication en faveur de la diversité. Citons le dispositif de la "Charte de la diversité », lancée en 2004 par deux entrepreneurs, Claude Bébéar et Yazid Sabeg3. Elle s'adresse aux entreprises soucieuses d'afficher une politique de ressources humaines se voulant non discriminatoire et valorisant la coexistence, en leur sein, de cultures, d'ethnies, de religions et de genres différents. Près de 3000 entreprises, en France, ont déjà signé cette charte qui semble avant tout répondre à une stratégie d'image et de performance économique. Cette prescription au pluralisme s'apparente en effet à une survalorisation de l'individualisme performatif : il s'agit de ne pas "gâcher des talents". La diversité est ici présentée comme un gage de réussite pour l'employé comme pour l'employeur.

Outil de communication externe des entreprises, la valorisation de la diversité concerne également le consommateur : communications institutionnelle et publicitaire sont indissociables. Après Benetton qui, dans les années 1980-1990, avait, non sans provocation, fondé sa campagne United Colors sur des principes civilisationnels de tolérance - qu'il réactualise aujourd'hui -, d'autres enseignes ont plus récemment formulé une promesse de bonheur, de partage et de cohésion sociale via la consommation et ce, grâce au thème de la diversité. La publicité s'est ainsi faite «militante pour permettre à l'entreprise d'acquérir une dimension universelle» (Tantet, 1992), et s'est simultanément voulue performative : non seulement la différence ne menacerait pas l'égalité, mais elle serait gage de richesse (humaine, axiologique, etc.).

McDonald's, en inventant la formule "venez comme vous êtes», la chaîne de supermarchés Casino, qui promet de «nourrir un monde de diversité» ou

2. La problématique de la redistribution et de la reconnaissance est posée par Nancy Fraser et expliquée par Fassin et Fassin (2006).

3. Ce dernier a ensuite été commissaire à la diversité et à l'égalité des chances auprès du gouvernement français de 2008 à 2011. 
encore L'Oréal, via son slogan «parce que je le vaux bien », font montre d'une stratégie de tolérance et d'ouverture à destination de leur clientèle, par le biais d'un vocable soigneusement choisi. Ces marques prétendent annoncer une société pacifiée parce que plurielle, une sorte de Tour de Babel inversée, laquelle suppose toutefois que l'on adhère à l'idéologie libérale dominante. L'enjeu de pouvoir et de contrôle est donc bel et bien présent. Sur ses affiches et dans ses spots télévisés, McDonald's met en scène plusieurs personnages, censés refléter la pluralité de la société française4, qui sont habillés et coiffés selon des moments divers de leur existence et de leur humeur. En dépit de leurs différences d'âge, d'origine, de genre ou de milieu social, ils ont en commun la fréquentation des restaurants «McDo », ce qui non seulement les fait se côtoyer, mais les rapproche et les relie les uns aux autres. Ce message idyllique et quasi religieux est d'autant plus ingénieux qu'il est destiné à communiquer sur une entreprise qui ne vend rien d'autre que des produits ultra-standardisés.

Pour Casino, «nourrir un monde de diversité » consiste à récupérer la multiplicité des habitudes alimentaires liées aux religions (casher, halal...), aux sensibilités, à la santé ou à l’hygiène de vie (produits végétariens, « light» ou "bio »), autrement dit aux différences des cultures entendues dans un sens très large, dans un seul but : développer ses ventes. La consommation garantirait donc l'égalité et le respect de tous, l'harmonie sociale : c'est le «panem» de «panem et circenses».

De son côté, L'Oréal, depuis une dizaine d'années, utilise l'image de différentes actrices et mannequins pour promouvoir ses produits cosmétiques. Celles-ci présentées comme reflétant une diversité physique - couleur de peau et d'yeux, type de cheveux, etc. - afin de s'adresser, symboliquement, à toutes les femmes, quelles qu'elles soient, en Europe, en Amérique du Nord, mais aussi dans un nombre croissant de pays d'autres continents (Asie, Afrique, etc.). Néanmoins, l'ensemble de ces égéries correspondent aux critères dominants de la beauté occidentale : elles sont jeunes, grandes, minces et les traits de leurvisage sont fins. La valorisation du pluralisme est ainsi particulièrement trompeuse ; elle s'apparente à un mythe dont la fonction est d'imposer un ordre social par la norme physique.

Même dans le marketing publicitaire, la diversité apparaît donc comme une opportunité politique tout autant qu'économique : dans une sorte de conjuration du repli communautaire comme du communautarisme, largement craints et dénoncés, l’idée véhiculée est celle que la société, devenue automatiquement, et sans combat, ouverte et tolérante, non seulement nous acceptera, mais nous valorisera tels que nous sommes. Dans un paradoxe qui n'est qu'apparent, la diversité est ici à la fois un élément de l’individualisme contemporain (le besoin

4. La campagne est déclinée sur le même mode dans les autres pays occidentaux, en particulier aux États-Unis. 
d'authenticité de tout un chacun) et une forme de résistance à ce dernier (le désir d'être accepté et respecté par autrui). En cela, elle prétend donner du sens. Cependant, la mise en valeur, par la publicité, d'une philosophie morale altruiste, de la confiance dans les spécificités de chacun vise avant tout à construire une identité forte pour la marque et à créer une relation émotionnelle avec l'individu qui n'est rien de plus qu'un consommateur. Or c'est aussi un électeur.

\section{La diversité comme outil de communication politique}

Depuis 30 ans, certains glissements sémantiques dans le vocable politique français sont un indice intéressant de l'évolution de la communication politique visà-vis des minorités : les expressions «issus de l'immigration » (années 1980), puis «minorités visibles» (années 1990) ont cédé le pas à celle d'«issus de la diversité » (années 2000), beaucoup moins stigmatisante et dans laquelle chacun (et pas seulement les immigrés et leurs enfants) peut se reconnaître. Les collectivités locales communiquent beaucoup sur et par la diversité, en particulier dans leur combat en faveur de l'égalité des chances. Nous avons discuté ailleurs de cette mise en récit du pluralisme culturel par la Ville de Paris (Naves, Pégard, 2009)5. Un autre exemple est celui de la signature, en 2009, d'un partenariat entre le conseil général de Seine-Saint-Denis et TF1, nommé «Convention médias et diversité », qui fait figure de code de bonne conduite réciproque. La chaîne de télévision s'engage en effet à former, voire à embaucher des jeunes gens demeurant dans le "93». Pour le département de Seine-Saint-Denis, c'est une occasion de présenter la pluralité des origines de ses habitants et le nombre important de jeunes adultes parmi eux comme une source de potentialités, et ainsi de gommer l'image de délinquance qui lui est attachée6.

Le « flyer» de l'édition 2011 montre le président de TF1 entouré de nombreux jeunes hommes et jeunes femmes de toutes origines «ethniques», souriants et serrés les uns contre les autres comme sur une photo de groupe dans une entreprise - qui rappelle la très récente campagne de la Société générale sur «l'esprit d'équipe»-, à l'image de ce que pourrait être la société française. Le slogan «pourquoi pas vous? ?, à l'instar du «venez comme vous êtes» de McDonald's - qui s'adresse aussi à ses employés -, est volontariste, rejette la fatalité, autrement dit adhère pleinement auxvaleurs libérales d'authenticité, de dépassement de soi et de performance.

5. Le journal municipal parisien Tous Parisiens, tous citoyens nous est ainsi apparu comme étant à la fois un outil de marketing territorial (Paris, ville cosmopolite et accueillante), de promotion de la citoyenneté et de l'intégration républicaines, et enfin d'encouragement à la pacification sociale grâce au pluralisme culturel et religieux.

6. Le forum d'aide à la recherche d'emploi pour les jeunes Parisiens «issus de la diversité », intitulé «Paris de la diversité et du premier emploi » et supervisé par la Ville de Paris depuis 2006, constitue une autre illustration intéressante. 


\section{Les discours politiques de rejet d'un vivre-ensemble harmonieux via le pluralisme}

La mise en politique des mots construit des récits et des contre-récits qui sont parfois portés, successivement, par les mêmes acteurs. De 2005 à 2008 environ, le gouvernement français a affiché un message d'émancipation et de démocratisation de l'accès aux ressources dans et par la diversité, et ce, dans le but de neutraliser les conflits sociaux, de faire retomber le climat de violence intervenu après les émeutes de l'automne 2005. Cela s'est en particulier traduit par des nominations de personnalités «issues de la diversité» à des postes clés (ministres, secrétaires d’État, préfets...). Par la suite, le message a néanmoins cédé le pas à un discours d'assignation « raciale», " ethnique » et religieuse dans un sens négatif, qui a stigmatisé certaines catégories de la population, au premier rang desquelles les musulmans et les Français d'origine étrangère. Le discours de tolérance culturelle a été de plus en plus fragilisé au profit d'une rhétorique du repli qui met en avant la défiance vis-à-vis de l'altérité.

L'extrême droite a choisi de dramatiser la différence culturelle, synonyme pour elle de menace pour l'identité et le vivre-ensemble français - mais aussi pour l'économie de notre pays, en période de crise. Le fantasme d'une pureté de la nation - voire de la « race » - française n'est pas nouveau (Girardet, 1990 ; Winock, 2004) mais il semble trouver un nouvel écho au regard de certaines mutations sociales, économiques et géopolitiques qui affectent l'Europe et la France. Depuis sa création, le Front national a alimenté le mythe de l'impossible coexistence pluriculturelle et plurireligieuse et du danger du métissage. Il assimile à l'islam des pratiques qui n'en sont que des aspects extrêmes, voire extrémistes (port de la burqa) ou contingents (prière dans la rue), les mettant sur le même plan que la construction de mosquées ou le rite halal. Ainsi, en réaction à la place, selon lui croissante, de l'islam dans notre pays et aux discours de la gauche et des associations antiracistes qui déplorent l'échec de la promesse républicaine d'intégration des immigrés et de leurs enfants, il construit, avec d'autres groupuscules de la droite radicale et nationaliste, sa propre rhétorique prétendument laïque et anti-communautariste, en usant des registres de la victimisation, de la provocation et de l'exagération (parlant entre autres d' "islamisation de la France »). Depuis 2009, l'extrême droite a multiplié les campagnes de communication en ce sens. Par exemple, des publicités pour l'enseigne de restauration Quick ont été fréquemment détournées : le groupe «Égalité et réconciliation» montre, sur une affiche, un hamburger traversé par une broche qui est en fait un minaret et qui est dessiné comme une arme de guerre : noir, long, pointu et fin, tel une lance ou un missile. Du sang coule du hamburger, synecdoque de l'animal tué selon le rite halal, mais il pourrait aussi être celui d'un être humain victime d'une guerre sainte. Sur une 
image, réalisée par le FN de Villeurbanne, un restaurant Quick se voit ajouter un minaret, surmonté du croissant de l'islam. Cette iconographie est reprise sur une autre affiche, que l'on doit à «Riposte laïque» : des Quick à minarets sont présents en divers points d'une carte de France, à côté de laquelle est dessiné le visage d'une femme caché par un voile intégral. Le slogan est : "Non à l'islam sexiste et séparatiste ». Ainsi mis en scène, les hamburgers halal, les minarets et la burqa deviennent le signe, au sens de Roland Barthes, d'une prétendue «islamisation», en soi systématique et belliqueuse, de la France. Dans ce cas, la diversité fait l'objet d'une narration de la peur, du danger et de la violence; la société française est décrite, par des images autant que par des mots, comme étant menacée par le pluralisme, y compris dans la consommation. C'est donc un discours politique exactement et délibérément contraire à celui des enseignes de consommation de masse décrites supra. Il y a comme un double effet de miroir et d'entraînement. On notera du reste que le Front national dénonce depuis quelques mois une prétendue alliance entre l'islam mondial et le capitalisme : son rejet de la diversité participe donc logiquement de sa critique du libéralisme économique et de ses valeurs individualistes qui - il est intéressant de le noter - étaient autrefois associés aux juifs.

Depuis quelques années, la diversité culturelle apparaît comme un outil du marketing économique comme politique, dont les usages varient en fonction du message que l'on souhaite véhiculer. Pour les uns, la société multiculturelle est gage de bonheur, de paix et de richesse, et la consommation comme le travail et la participation politique peuvent y contribuer; pour les autres, elle signifie la ruine d'une civilisation. Dans les deux cas, on se situe dans un récit mythologique dont l'important est finalement d'être le narrateur, peutêtre plus que l'acteur, tant la diversité in fine échappe au contrôle. En France, le multiculturalisme et l'anti-multiculturalisme ne sont-ils pas, avant tout, le produit de discours?

\section{Références}

AlBouY Serge, 1994, Marketing et communication politique, Paris, L'Harmattan.

BARTHES Roland, 1957, Mythologies, Paris, Le Seuil.

FASSIN Didier, FASSIN Éric éd., 2006, De la question sociale à la question raciale? Repré-

senter la société française, Paris, La Découverte.

FASSIN Éric, 2002, "La parité sans théorie. Retour sur un débat», Politix, vol.XV, n60, p.19-32.

Fou CAult Michel, 1969, L'archéologie du savoir, Paris, Gallimard.

GIRARDET Raoul, 1990, Mythes et mythologies politiques, Paris, Le Seuil.

Mercier Arnaud, Wolton Dominique éd., 2008, La communication politique, Paris, CNRS. 
Mesure Sylvie, Renaut Alain, 1999, Alter Ego. Les paradoxes de l'identité démocratique, Paris, Aubier.

NAves Marie-Cécile, PÉGARd Olivier, 2009, "“Tous Parisiens, tous citoyens”. Analyse d'une communication politique pour la promotion de la diversité à Paris », Lieux communs, $\mathrm{n}^{0} 12$, p. 77-90.

Perrineau Pascal éd., 2001, Les croisés de la sociétéfermée, La Tour d'Aigues, L'Aube.

SALMON Christian, 2008, Storytelling. La machine à fabriquer des histoires et à formater les esprits, Paris, La Découverte.

TANTET Marie, 1992, "La stratégie publicitaire de Benetton», Communication et langages, n०94, p. 20-36.

WINOCK Michel, 2004, Nationalisme, antisémitisme et fascisme en France, Paris, Le Seuil. 